\section{Rapporto sulla sessione ordinaria della Camera medica svizzera del 21 e 22 giugno 2000 a Berna}

\section{F.-X. Deschenaux, secretario generale della FMH}

Questa sessione della Camera medica rappresenta una pietra miliare nella storia della FMH, per essere la prima ad essere composta secondo il nuovo statuto della FMH. La presente sessione ha riservato ai 196 delegati presenti un ordine del giorno quasi titanico, con 89 proposte depositate all'inizio della seduta e comprendeva - oltre alle questioni amministrative abituali (rapporto annuale, conti della FMH per l'esercizio appena concluso e budget per l'anno seguente), le elezioni per il periodo amministrativo 2000-2003, la nuova sistematica dei titoli di specialista e i programmi di formazione, come pure lunghe discussioni relative all'obbligo a contrarre e gli ultimi sviluppi della TarMed. Come ci si può immaginare questi ultimi due punti hanno avuto una risonanza particolare in seguito ai recenti interventi - non si tratta certo di un caso - del Dipartimento federale dell'interno e del suo capo.

Le decisioni sono presentate nel presente verbale secondo l'ordine sequenziale sulla lista dei punti all'ordine del giorno.

\section{Comunicazione e costituzione dell'Ufficio}

La seduta è aperta alle 9.45, quando il quorum di 100 delegati è largamente raggiunto.

Gli scrutatori proposti dal presidente (i dott. A. Sury, B. Hug, A. Bacchetto, A. Froidevaux, C.Ehrler e M. Hug) sono accettati tacitamente dai delegati: si trattava degli scrutatori della prima giornata. Per la seconda gli scrutatori proposti all'inizio della seduta dal presidente e accettati tacitamente dai delegati sono stati i dott. Z. Schneider, C. Ehrler, A. Kubli Bauer, M. Schibli, M. Kondo Oestreicher e C. Brunner.

L'Ufficio incaricato di fare lo spoglio delle schede di voto per le elezioni amministrative 2000-2003 è costituito dai collaboratori della Segreteria generale diretti da C. Kreyden, questo affinché i delegati scrutatori possano partecipare ai dibattiti e alle decisioni che proseguono durante le operazioni di spoglio. Questo modo di procedere corrisponde ad una lunga tradizione che non viene rimessa in forse dai delegati.
Il presidente $\mathrm{H}$. H. Brunner precisa all'attenzione del verbale, per ora e per il futuro, la regolamentazione da rispettare per quel che concerne le persone accompagnatrici. Le organizzazioni di base (società cantonali di medicina) [qui di seguito: SCM], l'Associazione dei medici assistenti e capoclinica (ASMAC) sono state sinora autorizzate a farsi accompagnare da un consulente non medico che ha il diritto di prendere la parola sino a quando una mozione d'ordine non glielo neghi. Questo uso è mantenuto, ma il CC, rispettivamente il presidente, si riserva il diritto di esigere che eventuali accompagnatori non medici di società di disciplina medica (qui di seguito: SDM) siedano non nella sala, ma nelle tribune, e questo a causa del poco posto disponibile in seguito al grande effettivo di delegati presenti. A parte ciò, il CC si riserva sempre il diritto di invitare alle sedute della Camera medica, per il trattamento di un oggetto particolare, determinate persone esterne, la cui presenza risultasse necessaria.

Tenuto conto dell'impressionante lista di oggetti da trattare nel corso di questa sessione, il presidente lascia intendere che la riunione della prima giornata durerà molto probabilmente sino alle 18.30 circa, l'ora di chiusura del giorno seguente dipenderà dalla chiusura dell'ordine del giorno.

Conformemente al nuovo Regolamento d'esecuzione della FMH dell'8 aprile 1999 che si basa sull'articolo 35, cpv. 2 del nuovo statuto, d'ora in avanti verrà redatto un verbale delle decisioni, arricchito qui e là da qualche spiegazione supplementare, là dove necessario.

Il presidente costata, inoltre, che la convocazione e la lista dei punti all'ordine del giorno, compresa la pubblicazione nel Bollettino dei medici svizzeri (qui di seguito: BMS) sono state comunicate entro i termini. Tutte le proposte pervenute sino a lunedi sera sono state tradotte.

Su invito del presidente, l'amministratore di FMH Services, M. Baumgartner, spiega che il nuovo statuto di FMH Services permette anche a dei non membri della FMH di far parte di questa società, ragione per la quale non è più necessario tenere un'assemblea generale dei delegati di FMH Services prima della Camera medica ordinaria. L'assemblea dei delegati di FMH Services è ora sostituita da un'assemblea universale che prenderà le sue decisioni sotto forma di votazione generale, per la quale i membri riceveranno il materiale di voto prossimamente.

Il presidente conclude questo primo punto all'ordine del giorno rendendo omaggio alle consorelle e confratelli deceduti nel corso dello scorso esercizio. Ricorda in particolare la memoria del dott. Jörg Ammann, ex presidente della FMS, scomparso recentemente e di cui sottolinea i grandi meriti acquisiti con il suo eccezionale impegno in favore della causa dei confratelli chirurghi. Sottolinea che la sua lotta non si è accontentata solamente di difendere i loro onorari, in quanto la sua azione mirava molto più in alto e che rientrava nei suoi sforzi contro la svalutazione del loro statuto professionale. In questo senso merita il riconoscimento dell'insieme del corpo medico. 
Su invito del presidente, l'assemblea si alza e osserva qualche attimo di silenzio in memoria di tutti i suoi membri scomparsi.

\section{Rapporto annuale $1999 / 2000$}

Il rapporto annuale, elaborato dal presidente della FMH, dai membri del CC responsabili dei settori, dal segretario generale e dai diversi responsabili di divisione e dei servizi in seno alla Segreteria generale, è stato pubblicato dal BMS $n^{\circ} 23$ del 7 giugno 2000.

Il presidente lo completa con qualche osservazione retrospettiva e le prospettive per il periodo amministrativo che si apre a partire da oggi.

Dopo questa introduzione da parte del presidente, la parola non viene chiesta e il rapporto annuale viene allora approvato senza opposizione.

\subsection{Conti 1999 della FMH}

\subsubsection{Approvazione}

Il bilancio e i conti annuali della FMH per l'esercizio 1999 e il rapporto di verifica della fiduciaria PriceWaterhouseCoopers ad esso allegato sono approvati alla quasi unanimità, con un'astensione. L'eccedente delle uscite di Fr. 77744.98 , notevolmente inferiore ai Fr 429 000.- che erano stati previsti dal budget, è messo a carico del capitale proprio che ammonta cosi, al 31 dicembre 1999, a Fr. 4450873.69.

Vengono quindi approvati simultaneamente il bilancio e i conti d'esercizio al 31 dicembre 1999 del Fondo d'assistenza della FMH, come anche il bilancio e i conti d'esercizio al 31 dicembre 1999 del Fondo per l'incoraggiamento del perfezionamento professionale dei medici nei paesi in via di sviluppo.

\subsubsection{Scarico al Comitato centrale}

Lo scarico agli organi responsabili, per la loro gestione, viene votato a forte maggioranza, con un'astensione.

Proposta del dott. R. Streit, Società dei medici del canton Berna, al punto 3.1

La proposta, che riceve il sostegno incondizionato del dott. M. Battaglia, a nome dell'ASMAC, recita quanto segue: "Basandosi sui principi della contabilità analitica, il Comitato centrale è incaricato di dimostrare, alla Camera medica 2001, come le uscite e le entrate sono suddivise per unità di gestione. Si tratta anche di indicare il personale occupato per posizione spese."

Il presidente H. H. Brunner, a nome del CC, accetta questa proposta.

\subsection{Budget 2001 della FMH}

Il budget presentato alla Camera medica per l'anno 2001 presenta due aspetti: il budget generale per un totale di entrate di Fr. 12755000 .- e un totale di uscite di Fr. 12684 000.-, cioè un eccedente di entrate di Fr. $71000 .-$, da una parte, e dei contributi speciali e limitati nel tempo per finanziare le attività particolari, dall'altra. Come presentato, il budget generale si basa su una quota centrale di Fr. 480.- per i membri delle categorie 1 (medici con attività privata) e 2 (medici salariati che esercitano una funzione dirigente), di Fr. 240.- per i membri delle categorie 3 (medici salariati senza funzione dirigente e che non stanno seguendo un perfezionamento professionale FMH) e 4 (medici che seguono un perfezionamento professionale) e infine di Fr. 120.- per i membri delle categorie 5 (medici domiciliati che esercitano all'estero) e 6 (membri temporaneamente senza attività medica).

$\mathrm{Su}$ proposta del CC accettata tacitamente dalla Camera medica, si vota separatamente sul budget generale e sui contributi particolari.

Il budget generale non suscita nessuna discussione. $\dot{E}$ accettato a grande maggioranza meno un voto e tre astensioni.

I contributi speciali, invece, danno luogo ad una lunga discussione di cui riassumiamo qui le decisioni. L'importo dei contributi speciali inizialmente previsti dal CC ammonta a Fr. 120.- per tutti i membri e deve servire a finanziare i diversi compiti seguenti: Centro svizzero di farmacovigilanza: Fr. 5.-, per l'ultima volta nel 2001; contributo unico di Fr. 20.- nel 2001 allo scopo di finanziare lo sviluppo delle edizioni EMH; "Abbonamento HIN per tutti», da prelevare nel 2001, 2002 e 2003, al massimo Fr. 50.-; “Attività d'assistente nello studio medico» Fr. 25.- per le categorie di membri 1, 2, 3 e 4; infine Fr. 20.- per la fine dei lavori e l'introduzione della TarMed per le categorie di membri 1, 2, 3 e 4.

A coloro che, come il dott. R. Streit (BE), avevano desiderato che si procedesse il più possibile nel richiedere i fondi necessari integrandoli nel budget generale, il presidente giustifica il principio dei contributi speciali con l'argomento di avere una buona trasparenza e con il raggruppamento del finanziamento di compiti limitati nel tempo. Per quel che concerne i rappresentanti dell'ASMAC (dott. M. Battaglia e S. Stöhr), pur esprimendo delle riserve su alcuni argomenti (in particolare HIN), non contestano il principio di un finanziamento solidale, ma propongono che questo sia meglio modulato, nella stessa proporzione di quello tra le diverse categorie di quote. Detto ciò, il finanziamento di questi compiti non viene contestato da nessuno, alcuni delegati gli sono addirittura favorevoli, in particolare all'HIN.

Il presidente $\mathrm{H}$. H. Brunner propone allora una soluzione di compromesso a nome del CC. Propone la percezione di un contributo speciale ovvero di Fr. 100.- per tutti (variante I), oppure di Fr. 70.- per le categorie 3 e 4 e di Fr. 110.- per le categorie 1 e 2 (variante II). La variante scelta sarà allora opposta, in una votazione definitiva, alla proposta dell'ASMAC. Questo modo di fare viene accettato e il dott. P.-A. Schneider, dell'associazione dei medici del canton di Ginevra (AMG), che aveva presentato una proposta che richiede che il contributo per l'assistente nello studio medico sia ridotto di Fr. 25.- per passare a Fr. 10.-, ritira allora la sua proposta. Il presidente pre- 
cisa, prima della votazione, che gli importi raccolti a titolo di contributi speciali saranno allora attribuiti ai diversi oggetti tramite decisione del CC.

In una prima votazione con un risultato di stretta misura, la variante I prevale sulla variante II con 76 voti contro 75. In una seconda votazione che oppone la variante I alla proposta dell'ASMAC, prevale la variante I con 117 voti favorevoli, 49 contrari e 3 astensioni.

Non possiamo concludere questo capitolo senza citare anche un suggerimento del presidente della Società di medicina del canton San Gallo, il dott. R. Urscheler, che chiede che si organizzi ancora una volta, in occasione della prossima seduta della Conferenza dei presidenti, un sondaggio presso le SCM concernente l'incasso delle quote della $\mathrm{FMH}$, per sapere se devono procedervi le SCM oppure la Segreteria generale della FMH. Il suggerimento viene accettato.

\section{Elezioni per il periodo amministrativo 2000-2003}

4.1 Determinazione del numero di membri del CC per il prossimo periodo amministrativo

Questa questione mette in competizione delle proposte contraddittorie. Da una parte l'ASMAC e la Società medica di Basilea Città presentano delle proposte che mirano a mantenere l'effettivo del CC a 11 membri; dall'altra, il dott. A. Haefeli, presidente del VEDAG, aveva inizialmente presentato una proposta che chiede che i due seggi di lingua tedesca divenuti vacanti in seno al CC non siano più occupati, nel senso di una specie di moratoria. Il dott. Haefeli ritira tuttavia la sua proposta nel corso delle deliberazioni, tenuto conto che un seggio deve essere riservato per statuto all'Associazione dei medici principali di ospedali svizzeri (AMPOS) e per il fatto che anche coloro che oggi parlano a favore di un CC di 11 membri non si oppongono ad un principio di ulteriore riduzione, sempre che il CC presenti entro il 2001 un nuovo concetto per la direzione della FMH. Il dott. A. Haefeli si affianca quindi, senza nessuna restrizione, alla proposta del dott. R. Streit presentata qui sotto al punto 5 e dichiara che quanto attiene alla proposta del VEDAG, purché non concerna il nuovo periodo amministrativo, dovrà essere discussa al punto 5/cambiamenti dello statuto.

Altri delegati auspicano sin d'ora un effettivo ridotto a 9 membri. Si tratta quindi di decidere per una delle due tesi. In votazione i delegati decidono con 125 voti contro 48 e 4 astensioni, di rimanere per il periodo amministrativo 2000-2003 ad un CC composto da 11 membri.

\subsection{Elezioni del Comitato centrale della FMH}

Conferma dei membri attuali

Schede raccolte

Schede valide

Maggioranza assoluta
Sono rieletti:

VEDAG

dott. Hans Heinrich Brunner (LU) 165 voti

dott. Max Giger (ZH)

dott. Reto Laetsch (SG)

dott.ssa Ursula Steiner-König (BE)

150 voti

156 voti

155 voti

\section{SMSR}

dott. Claude Aubert (GE)

166 voti

152 voti

dott. Yves Guisan (VD)

158 voti

ASMAC

dott. Ludwig-Theodor Heuss (BS)

167 voti

Elezioni per i seggi vacanti:

ORDINE (OMCT)

Schede raccolte

175

Schede valide

174

Maggioranza assoluta

88

É eletto:

dott. Franco Muggli (TI)

174 voti

\section{AMPOS}

Schede raccolte

172

Schede valide

Maggioranza assoluta

168

85

É eletta:

dott.ssa Verena Briner, p.-d. (LU)

94 voti

Hanno raccolto voti:

dott. Yves Guisan (VD)

prof. Werner Straub (BE)

15 voti

1 voto

\section{Seggio restante}

Schede raccolte

Schede valide

161

Maggioranza assoluta

È eletta:

dott.ssa Susanna Stöhr (BS)

83 voti

Hanno raccolto voti:

dott.ssa Catherine Wehren (BS)

dott. Ludwig-Theodor Heuss (BS)

76 voti

2 voti

4.3 Elezione del presidente della FMH

Schede raccolte

Schede valide

163

Maggioranza assoluta

È eletto:

dott. Hans Heinrich Brunner

152 voti

Hanno raccolto inoltre voti:

dott. Claude Aubert

3 voti

dott. Yves Guisan

dott. Ludwig-Theodor Heuss
5 voti

3 voti 


\subsection{Elezione dei due vice presidenti della FMH}

Elezioni per alzata di mano

Facendo seguito ad un suggerimento proveniente dall'assemblea, il presidente H. H. Brunner mette dapprima in votazione la questione di sapere se i delegati accettano che a partire da subito le elezioni siano effettuate per alzata di mano. I delegati approvano questo modo di procedere con 147 voti favorevoli, 19 contrari e 3 astensioni, cioè con una maggioranza superiore ai $3 / 4$ (127) richiesti dallo statuto.

Partecipano all'elezione dei vice presidenti: 170 delegati

Sono eletti:

dott.ssa Ursula Steiner-König (nuova)

dott. Yves Guisan (sinora)

152 voti

143 voti

4.5 Elezione del presidente della Commissione per il perfezionamento professionale e l'aggiornamento (CPA)

Partecipano all'elezione del presidente della CPA: 159 delegati

È eletto:

dott. Max Giger (nuovo)

150 voti

\subsection{Elezione della Commissione delle finanze della FMH}

Si procede a questa elezione «in globo", sia per il rinnovo dei due membri uscenti, i dott. O. Kappeler (TG) e A. Sury (TI), come anche per il nuovo membro proposto dalla SMSR, il dott. Pierre Guillaume (FR). Sono tutti e tre eletti con 171 voti.

\subsection{Elezione dell'Ufficio del Consiglio svizzero di deontologia (CSD)}

Anche a questa elezione si procede "in globo", per acclamazione, poiché non si è presentato nessun altro candidato oltre a quelli proposti della VEDAG, dalla SMSR e dall'ORDINE. Si tratta del dott. Sergio Luisoli (TI), sinora, del prof. Markus Heinz Knoblauch (ZH), (sinora) e del dott. Dominique Haefeli (NE), nuovo.

Nota bene: poiché il vecchio e i nuovi membri non hanno ancora avuto l'occasione di incontrarsi, l'elezione del presidente della CSD viene posposta sino alla prossima sessione della Camera medica.

4.8 Comitato consultivo per le negoziazioni TarMed Il CC aveva proposto alla Camera medica di costituire un Comitato consultivo di 10 membri, cioè 4 per la Foederatio Medicorum Scrutantium (FMS), 4 per il Collegio dei medici di famiglia (CMPR) e 2 per la Foederatio Medicorum Curantium (FMC).

Una proposta congiunta della Società svizzera di psichiatria e psicoterapia e della Società svizzera di psichiatria e psicoterapia per $i$ bambini e gli adolescenti chiede che la composizione di questo comitato consultivo sia portata a 12 membri, affinché i due seggi supplementari possano esservi riservati alla SSPP.

Votazione: la proposta degli psichiatri è accettata con 138 voti favorevoli, 21 contrari e 8 astensioni.
Nel frattempo la Camera medica prende conoscenza delle proposte della FMS, del CMPR, della FMC e della SSPP e le accetta tacitamente senza nessun'altra controproposta. In questo Comitato consultivo di 12 membri sederanno:

Per la FMS: i dott. Beat Meister (BE), Alexander Eijsten (ZH), Walter P. Gartenmann (ZH) e Roberto Tartini (ZH). Quattro supplenti sono stati anche previsti dalla FMS in caso di impedimento degli eletti: l'avv. A. Bernath e i dott. Christoph Krayenbühl, Rolf Steiner e Josef Hug;

Per il CMPR: i dott. Christoph Franke (SG), Niklaus Hasler (ZH), Juerg Pellaton (ZH) e Emil Simon (VD);

Per la FMC: dott. Markus Heitz (ZH) e Andreas Wüest (ZH);

Per la SSPP: i dott. Heiner Lachenmeier (ZH) e Urs Fromm (AG).

Prima di lasciare il capitolo delle elezioni, il presidente $\mathrm{H}$. H. Brunner vuole rendere omaggio ai tre membri del CC che ci lasciano alla fine del periodo amministrativo appena conclusosi e ringraziarli, sottolineando i loro meriti e i compiti svolti al servizio della FMH e dell'insieme del corpo medico nel corso di numerosi anni d'attività. Torneremo ulteriormente sul soggetto in un articolo del BMS. Ognuno dei membri dimissionari si rivolge quindi personalmente all'assemblea e viene applaudito dai delegati.

\section{Cambiamento dello statuto}

Il dott. A Haefeli, a nome del VEDAG, riprende qui l'idea già evocata in una proposta al punto 4.1 (vedi sopra) con la quale il Comitato centrale è invitato a presentare alla Camera medica un progetto di ristrutturazione e di revisione del suo modo di lavorare, tenendo conto della riduzione del numero di membri, della rappresentazione appropriata delle regioni linguistiche, della non eleggibilità dei rappresentanti di gruppi d'interesse e della divulgazione dei legami potenzialmente problematici, dell'aumento del tasso di professionalità, del ruolo più attivo del CC e del suo presidente quando si tratta di scegliere i candidati e infine della preparazione dei candidati su un periodo più lungo per assumere la loro funzione, tenuto conto del cambiamento d'attività professionale. Calendario previsto: prima versione da presentare alla Camera medica del giugno $2001 \mathrm{e}$ concetto dettagliato nel 2002, affinché i candidati al CC del prossimo periodo amministrativo possano essere preparati sufficientemente presto.

Al momento della votazione, la proposta del $V E D A G$, alla quale il CC non si è opposto, è accettata con 132 voti contro uno, con 17 astensioni.

Una proposta n. 3 al punto 5 dell'ordine del giorno, presentata a nome della Società dei medici del canton di Berna dal dott. R. Streit, mira in sostanza agli stessi obiettivi di quelli della proposta del VEDAG, ma chiede in più che «un comitato ampliato 
con competenze precise [venga] a porsi tra il piccolo comitato e la Camera medica".

Dopo un breve dibattito nel quale le persone che sono intervenute esprimono nella grande maggioranza il loro scetticismo a proposito di questo genere di istituzione, si passa alla votazione. La proposta del dott. R. Streit viene respinta con 102 voti, contro 49 e 21 astensioni.

Una proposta congiunta della SSMG e della SSMI $n$. 1 al punto 5 dell'ordine del giorno mira a che "la FMH [sia] incaricata di formulare dei criteri appropriati, che garantiscano la proporzionalità della ripartizione dei seggi delle società di disciplina medica alla Camera medica in conformità al nuovo statuto della FMH".

Questa proposta è accettata con 134 voti favorevoli, 25 contrari, e 8 astensioni.

Questo risultato porta il dott. A. Kubli a ritirare la proposta $n .2$ al punto 5 presentata congiuntamente dalle due società di psichiatria e psicoterapia (adulti/ bambini e adolescenti) e che persegue lo stesso obiettivo.

\section{Perfezionamento professionale}

\subsection{Nuova sistematica dei titoli di specialista/ programmi di formazione}

\subsubsection{Revisione dei titoli di specialista}

Prima di entrare nei dettagli delle deliberazioni e delle decisioni, il dott. R. Salzberg, presidente uscente della CPA, presenta l'ambito del dibattito, quanto ci sia in gioco e le decisioni. Ricorda che nel 1994, di fronte alla profusione dei nuovi titoli, è stata decretata una moratoria. Ne è risultata una revisione del Regolamento per il perfezionamento professionale (RPP) nel 1997 che ha condotto alla creazione di nuovi titoli di specialista e alla creazione di formazioni approfondite, di attestati di perfezionamento professionale e di certificati di attitudine tecnica che dovrebbero sostituire e integrare le vecchie sottospecializzazioni che sono state abolite. Da allora, sono stati creati nuovi titoli di specialista come anche delle specializzazioni approfondite. La Camera medica ha sviluppato un uso che prevede che il perfezionamento professionale doveva durare al massimo 6 anni, che ci si doveva mostrare restrittivi con la creazione di nuovi titoli e che questi ultimi dovrebbero contare al minimo su 3 anni di perfezionamento professionale specifico nella specializzazione. È stato inoltre deciso di adottare un modello secondo il quale il titolo di specialista +3 anni di formazione complementare nella disciplina potrebbe condurre ad una formazione approfondita (esempio: la neonatologia).

Dobbiamo discutere oggi di due generi di problemi che non si iscrivono in questo concetto, quello della neuroradiologia e quello delle vecchie sottospecializzazioni della pediatria. Il dott. R. Salzberg ricorda quelle che sono le considerazioni di principio da ritenere per le decisioni da prendere da parte della
Camera medica: un titolo di specialista serve ad attestare che un perfezionamento professionale strutturato e controllato sia compiuto conformemente al regolamento in una disciplina clinica o non clinica della medicina, mentre, come lo indica il suo nome, una formazione approfondita attesta che la formazione postgrade è stata spinta più avanti in un settore particolare di una disciplina. I gruppi responsabili di queste formazioni approfondite sono rappresentate nella CPA e possono farvi valere i loro desideri; invece questi gruppi non sono rappresentati come tali alla Camera medica e devono allora passare dalle loro società madri per presentarvi le loro domande (esempio: i neonatologhi devono incaricare la Società svizzera di pediatria, ecc.). D'altra parte dobbiamo tener conto della pratica seguita in materia dai medici della Comunità europea ed esaminare il modo in cui questi settori di specializzazione vi sono regolati. Infine, ogni società di disciplina che gestisce una specializzazione ha l'obbligo di mantenere aggiornato il suo programma di formazione postgrade e di adattarlo all'evoluzione della medicina, deve organizzare un esame per l'ottenimento del titolo di specialista e garantire il perfezionamento e la qualità delle prestazioni dei suoi membri.

Spetta ora alla Camera medica decidere se vuole creare una specialità o una formazione approfondita, mentre fissare i programmi rientra nelle competenze del CC. Se una società di specialisti sottopone un programma per la creazione di un titolo di specialista e c'è unità di visione in seno alla CPA, non si discute su questa proposta. Invece, se delle proposte sono presentate a proposito di questi programmi, verranno dibattute alla Camera.

Si passa quindi alla discussione di dettaglio.

In una proposta $\mathrm{n} .1$ al punto 6, il dott. M. Battaglia, a nome dell'ASMAC, propone una modifica dell'ordine del giorno che consiste nel trattare dapprima al punto 6.1 la revisione del RFF (prevista nell'ordine del giorno al punto 6.2) e che si tratteranno solo in un secondo tempo gli oggetti relativi alla nuova sistematica dei titoli di specialista/programmi di perfezionamento (previsti al punto 6.1). Il dott. R. Salzberg, a nome del CC, si oppone a questa modifica delle regole del gioco.

Al momento della votazione la proposta dell'ASMAC è respinta con 124 voti contrari, 30 favorevoli, 10 astensioni. Si proseguono quindi le deliberazioni conformemente all'ordine del giorno previsto dal CC.

6.1.1.1. Anestesiologia

La Camera deve occuparsi di una proposta depositata dal dott. M. Battaglia, presentata a nome dell'ASMAC, che si oppone al fatto che la durata del perfezionamento professionale in anestesiologia passi da cinque a sei anni.

Al momento della votazione la proposta del Comitato centrale (sei anni) è accettata con 110 voti favorevoli, 39 contrari, e 18 astensioni, cosa che quindi significa il rigetto della proposta dell'ASMAC. 
6.1.1.2 Chirurgia plastica, ricostruttiva ed estetica Ricordiamo qui che questo punto è stato ritirato dall'ordine del giorno su richiesta stessa della società di disciplina; questa aveva in effetti constatato che il termine qualitativo "estetica" che voleva aggiungere al titolo della specializzazione non figura neanche nei corrispondenti titoli stranieri.

\subsubsection{Creazione di titoli di specialista}

6.1.2.1 Neuroradiologia; decisione della CPA dell'11 maggio 2000

Il dott. M. Giger, nuovo presidente della CPA, introduce la problematica che noi presentiamo qui in modo riassunto.

La controversia che divide la Società svizzera di radiologia medica (SSRM) e la Società di neuroradiologia sta nel fatto che la prima chiede la creazione di formazioni approfondite per la neuroradiologia, mentre la seconda chiede la creazione di un titolo specifico per la sua disciplina, facendo valere (il suo rappresentante, il dott. 0 . Schubiger, lo ricorderà davanti alla Camera) che non sarebbe più possibile coprire i due settori e che si potrebbe temere che questa specializzazione, se le fosse tolto il titolo, sparirebbe. La CPA e la Conferenza dei presidenti propongono alla Camera medica la creazione di due formazioni approfondite, una di "neuroradiologia dignostica", l'altra di "neuroradiologia interventiva".

$\mathrm{Nel}$ corso della discussione accanita che si sviluppa sull'argomento e che vede i partigiani delle due tesi scontrarsi, il prof. F. Follath (SSMI) presenta una proposta che mira a che le sue società riprendano le discussioni in vista di presentare ad una prossima $\mathrm{Ca}$ mera medica un programma di perfezionamento professionale che permetta di rilasciare un doppio titolo in radiologia e neuroradialogia. Il dott. M. Giger si oppone a questa proposta, ricordando il pericolo che una tergiversazione del genere rappresenterebbe per gran parte degli altri settori del regolamento per il perfezionamento professionale in radiologia (radiologia pediatrica, radio-oncologia) e dichiara che è imperativo che la Camera medica decida oggi sulla controversia. Prima di passare alla votazione, il prof. P. Schnyder (CHUV), in quanto titolare di una cattedra di radiologia e rappresentante svizzero nelle più alte istanze europee della specialità, si oppone risolutamente alla proposta della Società svizzera di neuroradiologia, facendo valere tra l'altro le conseguenze finanziarie che la creazione di un nuovo titolo in neuroradiologia causerebbe per gli istituti di formazione, i pericoli di secessione che implicherebbe nella disciplina e il fatto che al momento solamente due paesi europei (Svezia e Portogallo) conoscono un titolo del genere.

Al momento della votazione la proposta del prof. F. Follath è respinta con 130 voti contrari, 24 favorevoli e 4 astensioni.

Si può quindi passare alla votazione sulla proposta del CC. Con 147 voti favorevoli, 14 contrari, i delegati optano per la creazione delle due formazioni approfondite e, quindi, per l'ammissione della Società svizzera di neuroradiologia nella CPA.

\section{Sottodiscipline pediatriche}

Si tratta anche qui di decidere tra due alternative, titolo di specialista o formazione approfondita. Come precisa il dott. H. H. Brunner, l'opzione scelta deve valere per tutte le forme di sottodiscipline enumerate e non si deve assolutamente trattare le une in modo differente dalle altre. Di conseguenza propone che si voti su tutte queste sottospecializzazioni «in globo" a meno che la discussione non sia richiesta su una sottospecializzazione particolare.

Dopo un dibattito in cui sono state difese le varie tesi, in particolare dai rappresentanti delle diverse società che gravitano nel settore della pediatria che parlano a favore della creazione dei titoli di specialista, si vota. Il presidente constata per prima cosa che nessuno si oppone ad una procedura di voto "in globo" per tutte le sottodiscipline in discussione. La Camera medica si pronuncia in favore della soluzione delle formazioni approfondite con 114 voti favorevoli, 43 contrari e 7 astensioni.

Di conseguenza questa soluzione sarà applicata d'ora in avanti alle sottospecializzazioni seguenti:

6.1.2.2 Endocrinologia-diabetologia pediatrica

6.1.2.3 Gastroenterologia pediatrica

6.1.2.4 Cardiologia pediatrica

6.1.2.5 Nefrologia pediatrica

6.1.2.6 Neurologia pediatrica

6.1.2.7 Oncoematologia pediatrica

6.1.2.8 Pneumatologia pediatrica

\section{Altri programmi di perfezionamento}

I programmi di formazione postgrade che seguono sono stati discussi solamente se erano l'oggetto di una controproposta scritta alla proposta del CC (come è stato il caso per il punto 6.1.2.13); altrimenti le proposte del CC sono considerate accettate. La Camera medica non si oppone a questo modo di procedere.

Di conseguenza la soluzione senza discussione si applica alle specializzazioni seguenti:

6.1.2.9 Allergologia e immunologia clinica: creazione di un titolo di specialista FMH

6.1.2.10 Medicina del lavoro: creazione di un titolo di specialista FMH

6.1.2.11 Endocrinologia-diabetologia: creazione di un titolo di specialista FMH

6.1.2.12 Ematologia: creazione di un titolo di specialista FMH

6.1.2.13 Medicina intensiva: la Camera deve occuparsi di una proposta presentata dall'ASMAC che si oppone alla creazione di un titolo, considerando che questo titolo non ha senso, tenuto conto della vasta formazione postgrade di base che questa specializzazione richiede in altre discipline cliniche. Il dott. R. Stocker, per la Società svizzera di medicina intensiva, sostenuto dal dott. B. Meister (anestesiologia), parla a favore del rilascio del titolo, ricordando l'effettivo rispettabile di «intensivisti» (250 e 30 in formazione postgrade), i 60000 interventi specifici per anno e la preoccupazione di non perdere il personale infermieristico che lavora nei 60 servizi di medicina intensiva. 
Si passa alla votazione dopo che il presidente $\mathrm{H}$. $\mathrm{H}$. Brunner ha spiegato che i voti di coloro che rifiuteranno la proposta del CC (creazione di un titolo di specialista in medicina intensiva), conteranno a sostegno della creazione di una formazione approfondita, conformemente alla proposta dell'ASMAC.

Alla votazione, la proposta del CC viene respinta con 78 voti contrari, 65 favorevoli e 9 astensioni. Ne consegue che i delegati si sono pronunciati per la creazione di una formazione approfondita.

\section{Osservazione importante:}

Appena noto il risultato, il dott. R. Stocker solleva la questione delle formazioni approfondite per 7 settori di discipline differenti. Il dott. R. Salzberg riconosce l'esistenza di un reale problema. Alla CPA, e anche al $\mathrm{CC}$, ci si dovrà rimettere al lavoro, e passare eventualmente ancora una volta davanti alla Camera medica.

6.1.2.14 Farmacologia e tossicologia clinica: creazione di un titolo di specialista FMH

6.1.2.15 Nefrologia: creazione di un titolo di specialista FMH

6.1.2.16 Oncologia medica: creazione di un titolo di specialista FMH

6.1.2.17 Pneumologia: creazione di un titolo di specialista FMH

6.1.2.18 Radiologia: la Camera è chiamata a decidere qui su una controversia relativa alla durata del perfezionamento professionale, 6 anni come lo proponeva il CC o solamente 5 anni come lo chiedeva l'ASMAC. Al voto, la proposta del CC ha la meglio con 100 voti favorevoli, 40 contrari e 14 astensioni. 6.1.2.19 Medicina nucleare: creazione di un titolo di specialista FMH

6.1.2.20 Radioencologia/radioterapia: controversia identica sulla durata della formazione postgrade su cui decidere: 6 anni come preconizzava la proposta del CC e 5 anni chiesti dall'ASMAC. Votazione: viene accettata la proposta del CC con 116 voti favorevoli, 25 contrari e 11 astensioni. La creazione del titolo di specialista FMH non viene rimessa in questione.

\subsubsection{Creazione di formazioni approfondite}

6.1.3.1 Neuroradiologia diagnostica (per la radiologia): creazione di una formazione approfondita ed ammissione della società di disciplina nella CPA.

6.1.3.2 Neuroradiologia interventiva (per la radiologia): creazione di una formazione approfondita e ammissione della società di disciplina nella CPA.

6.1.3.3 Radiologia pediatrica (per la radiologia): creazione di una formazione approfondita ed ammissione della società di disciplina nella CPA.

6.1.3.4 Medicina della riproduzione e gineco-endocrinologia (per la ginecologia e ostetricia): questo oggetto si iscrive in un contesto particolare che merita qualche spiegazione. Il Consiglio federale (CF) deve prossimamente far entrare in vigore la legge federale sulla procreazione assistita adottata dal Parlamento nel dicembre 1999. Questa legge prevede che i medici che praticano queste tecniche debbano ottenere un'autorizzazione. Piuttosto che sia la Confedera- zione a fissarne le esigenze, esiste una soluzione secondo la quale la FMH le definisce nell'ambito del suo $\mathrm{RPP}$, con la creazione anticipata di una formazione approfondita. L'Ufficio federale della giustizia propone al CF di far riferimento al regolamento della FMH nell'ordinanza d'applicazione. Questo spiega la fretta e la pressione alle quali siamo sottoposti per quanto concerne questa questione.

Questa pressione è considerata inammissibile dall'ASMAC, che in un primo tempo e senza opporsi al principio stesso della creazione di una formazione approfondita, aveva deposto una proposta di non entrata in materia. Questa è stata ritirata nel corso del dibattito, dopo che è stato assicurato che il programma (non contestato) di formazione approfondita per questo settore, presentato nell'ambito del programma per il titolo di ginecologia e ostetricia nel giugno 1999, non aveva subito nessuna modifica.

La Camera può così accettare la proposta del CC di creare una formazione approfondita in medicina della riproduzione e in gineco-endocrinologia. Si prende atto del fatto che le questioni tecniche di dettaglio che potrebbero risultare da questa decisione anticipata in rapporto al titolo di base ancora invariato dovranno essere regolate tra la SDM interessata, la CPA e il CC.

\subsubsection{Creazione di attestati di formazione complementare}

6.1.4.1 Medicina di immersione (SSMS): creazione di un attestato di formazione complementare in medicina di immersione (SSMS).

\subsubsection{Creazione di certificati d'attitudine tecnica}

6.1.5.1 Radiologia a forti dosi: creazione dei certificati di attitudine tecnica per esami radiologici a forti dosi.

6.1.5.2 Laboratorio (CMPR): creazione di un certificato di attitudine tecnica.

6.1.5.3 Laserterapia della pelle e delle mucose cutanee (FMS): creazione di un certificato di attitudine tecnica.

\subsection{Revisione del Regolamento}

per il perfezionamento professionale (RPP)

Prima di passare alla votazione sull'insieme del RPP, la Camera deve occuparsi di una proposta del dott. M. Battaglia, presentata a nome dell'ASMAC, che chiede che le commissioni di ricorso si compongano di 5 membri, e cioè di un legale indipendente, 2 membri del CC, un membro di una SCM e un membro dell'ASMAC.

Da parte de CC si fanno notare le buone esperienze fatte con una commissione di 3 membri e la preoccupazione di un'amministrazione con pochi oneri.

In votazione la proposta del CC su questo punto preciso è accettata con 115 voti favorevoli, 19 contrari e 5 astensioni.

$\mathrm{Su}$ richiesta del dott. R. Salzberg, la Camera procede ad un voto formale sul RPP nel suo insieme, dopo tutte le decisioni di dettaglio che la concernono, no- 
nostante che a partire dal 1 gennaio 2001, gli succederà una legge federale.

In votazione, il RPP viene approvato a grande maggioranza con 138 voti favorevoli, 2 contrari e 6 astensioni.

\subsection{La Legge sull'esercizio delle professioni} mediche: rilascio di titoli federali di formazione postgrade a medici senza titolo

All'articolo 24, cpv. 3 della Legge sull'esercizio delle professioni mediche, il legislatore federale ha deciso di rilasciare, a tutti i medici senza titolo, un titolo postgrade corrispondente alla loro formazione pratica e teorica tra i titoli figuranti nell'ordinanza d'esecuzione della legge. Questa disposizione è necessaria quando l'esercizio della professione medica a titolo indipendente non sarà più possibile senza titolo postgrade federale. L'Ordinanza propone, quindi, due varianti sulle quali - anche se la decisione finale spetta al Consiglio federale - il CC desidera disporre del parere consultivo della Camera.

La variante I, che presenta due sottovarianti, chiede la verifica completa del curriculum di formazione. Nella sottovariante 1 , un anno di formazione postgrade mancante potrebbe essere sostituito da un anno di attività in uno studio medico, mentre la sottovariante 2 permetterebbe di compensare sino a tre anni di formazione postgrade mancanti con un'attività in studio.

La variante II, che tiene conto dei desideri della SSMG, faciliterebbe il rilascio di un titolo di specialista in medicina generale sempre che il candidato possa attestare due anni di formazione postgrade valida.

Il dott. R. Hohendahl, della Foederatio Medicorum Practicorum FMP, chiede alla Camera medica di incaricare il CC di continuare a negoziare (con l'UFSP) ai sensi della sottovariante 2 .

$\mathrm{Al}$ termine di un lungo dibattito la proposta della FMP è sottoposta a votazione: viene rifiutata con 91 voti contrari, 37 favorevoli e 9 astensioni.

\section{Ammissione della Società svizzera dei chiro-} pratici in quanto $\mathrm{IV}^{\circ}$ società fondatrice della Cassa di compensazione dei medici, dentisti e veterinari (Cassa AVS n. 28).

Questo punto dell'ordine del giorno è trattato in presenza della signora Franziska Iseli, notaio di Berna.

Il segretario generale spiega brevemente il contesto della proposta. La parola non viene richiesta. Il presidente chiede allora all'assemblea, a nome del CC della FMH, di accettare la domanda della Società svizzera dei chiropratici di essere ammessa come $\mathrm{IV}^{\circ}$ società fondatrice nella Cassa di compensazione AVS n. 28, a partire dal 1 gennaio 2001. Messa in votazione questa proposta ottiene 161 voti favorevoli, 0 contrari e 2 astensioni. La maggioranza qualificata dei ${ }^{3}{ }_{4}$ dei voti espressi (123) è raggiunta e la proposta è cosi accettata.

\section{Codice di deontologia FMH / revisione parziale}

In una proposta presentata dal prof. L. von Laer a nome della Società basilese di medicina, si chiede che la serie di sanzioni previste all'articolo 47, cpv. 1 del Codice di deontologia della FMH del 12 dicembre 1996 sia arricchita, a partire dal 1 gennaio 2001, dalla possibilità di incoraggiare e sostenere i medici interessati mediante una supervisione (lett. h nuova). Il prof. L. von Laer fa notare che un tal modo di fare sarebbe in certi casi (abusi sessuali di pazienti) più efficace delle sanzioni puramente penali.

Il presidente $\mathrm{H}$. H. Brunner non si oppone a questa proposta che la Camera medica accetta tacitamente. $\grave{E}$ quindi trasmessa ai servizi competenti per esecuzione.

\section{Legge sui prodotti terapeutici (informazione orale)}

La legge che è stata trattata al Consiglio nazionale senza che si sia tenuto conto dei desideri da noi espressi, si trova attualmente nella commissione ad hoc del Consiglio degli Stati: è qui, quindi, che dovremo fare agire i nostri postulati (soluzione liberale per la distribuzione dei medicinali, affidandola a tutte le persone - medici inclusi - che hanno competenza e soluzione ragionevole per quel che concerne la sterilizzazione). Conviene ricordare qui che il CC ha ricevuto la competenza dalla Camera medica di lanciare un'iniziativa nel caso in cui l'evoluzione della questione lo rendesse necessario. D'altra parte, ci rimane anche la possibilità di un referendum contro la legge. Ricordiamo, infine, il suggerimento del dott. A. Haefeli che chiede al CC, all'intenzione delle SCM, uno studio che riassumi quello che dal punto di vista della FMH deve assolutamente trovarsi in questa legge; il presidente $\mathrm{H}$. H. Brunner ne prende nota a nome del CC. Affare da seguire.

\section{Obbligo a contrattare (informazione orale)}

Il dott. R. Streit, a nome della Società dei medici del canton Berna, chiede in una proposta al punto $10 \mathrm{del}$ l'Ordine del giorno che sia condotto "un dibattito di fondo sulla situazione della politica della sanità, tenendo conto delle dichiarazioni della consigliera federale R. Dreifuss al Consiglio degli Stati secondo le quali il valore del punto dovrebbe essere di 80 centesimi. Si tratta anche di evocare la proposta del Dipartimento federale dell'interno relativo alla sospensione dell'obbligo a contrattare nell'assicurazione sociale di base." Questa proposta è accetta alla quasi unanimità, con qualche voto contrario isolato.

Si apre quindi un lungo dibattito che illustra, come se ce ne fosse bisogno, il grado di esasperazione al quale è giunto il corpo medico riunito in seno alla FMH. Parole dure vengono pronunciate contro la consigliera federale R. Dreifuss, per il suo nuovo tentativo di fare pressione sui nostri dibattiti come anche 
per essere uscita dalla riserva che avrebbe dovuto osservare in quanto futuro "giudice di ultima istanza" chiamato a pronunciarsi sulla TarMed, al punto da perdere qualsiasi credibilità agli occhi della Camera medica per quel che concerne la sua imparzialità.

Per quel che concerne più in particolare la sospensione dell'obbligo a contrarre, la Camera è pronta a convenire una limitazione dell'obbligo a contrattare allo scopo di lasciare i medici migliori lavorare al servizio dei pazienti dell'assicurazione sociale e ricorda le sue proposte in materia inviate all'UFAS e al Concordato degli assicuratori svizzeri malattia. La $\mathrm{FMH}$, invece, non temerebbe in alcun modo un referendum se la riforma della legge fosse quella proposta dal Dipartimento federale dell'interno.

Per quel che concerne la TarMed e senza pregiudicare le decisioni ancora da prendere al punto 11 qui sotto, i delegati danno l'istruzione ai delegati alle negoziazioni di non accordarsi su un valore di punto inferiore a Fr. 1.- al momento delle negoziazioni finali.

Al termine della discussione, il presidente $\mathrm{H}$. $\mathrm{H}$. Brunner ne riassume i punti principali e informa la Camera che ne sarà fatto un comunicato stampa. $L a$ Camera accetta senza discussione sia il riassunto steso dal presidente sia il principio di un comunicato.

Nota bene: questa decisione è stata eseguita la sera stessa mediante la pubblicazione di due comunicati stampa, "Il corpo medico contro i tentativi di intimidazione del DFI" e "Soppressione dell'obbligo a contrattare: la FMH pensa al referendum».

\section{TarMed}

Il presidente H. H. Brunner deve fornire delle risposte ai rappresentanti della FMS (C. Krayenbühl e F. A. Bernath) sulle diverse questioni relative agli studi sul terreno (si è rinunciato agli studi pilota, considerati gli alti costi previsti), agli studi del cesto tipo (dovrà rimanere uno strumento di pilotaggio ad uso interno) come anche sulla perizia giuridica indipendente concernente i diritti e gli obblighi delle diverse persone intervenute nell'introduzione della TarMed (reticenza dei giuristi competenti a impegnarsi e interesse più che misurato in seno alla TarMed). Le prospettive sono migliori per quel che concerne il mandato da affidare ad un istituto neutrale straniero sulla questione relativa alla realizzazione della struttura tariffaria secondo le regole della gestione aziendale.

Gli interpellanti si dichiarano soddisfatti di queste risposte.

\subsection{Revisione 2000}

La Camera prende conoscenza dei risultati della revisione 2000 come anche del relativo commento che non si presta assolutamente ad essere esposto nel BMS, cioè sulla piazza pubblica.

\subsection{Modello tariffario della FMS}

La Camera prende anche conoscenza del commento scritto a proposito di questo oggetto come anche delle spiegazioni complementari fornite nel corso del di- battito dal presidente, spiegazioni che servono da risposta ad una proposta della FMS (n. 2 al punto 11.2 dell'ordine del giorno) che chiede che il modello FMS sia introdotto prima della messa in vigore della TarMed.

\subsection{Struttura tariffaria TarMed alfa 3.0/ precontratto}

Si ricorderà qui che la versione alfa 3.0 della struttura tariffaria è in via di elaborazione e che si basa sulla revisione 2000 e conterrà tutte le proposte e modifiche trattate sino al 27 maggio 2000. Questa versione costituirà la base delle negoziazioni per la tariffa medica AINF/AM/AI, per il catalogo delle prestazioni ospedaliere come anche per le tariffe cantonali. Una versione alfa $3 i$ sarà pubblicata ulteriormente; terrà conto solo delle modifiche di forma e di tipo redazionale. Sarà inviata a tutti i membri della FMH sotto forma di CD-ROM e sarà l'oggetto di una votazione generale. Poiché le proposte ci sono pervenute dopo il 15 aprile 2000 sono rinviate alla $I^{\circ}$ revisione della versione 3.0.

Una proposta (n. 2 al punto 11.3) della Società svizzera di psichiatria e di psicoterapia come anche della Società svizzera di psichiatria e psicoterapia del bambino e dell'adolescente prega il CC «di adottare le misure appropriate perché si riprendano in considerazione gli scopi iniziali del GRAT, cioè tenere meglio conto delle prestazioni intellettuali e emotive [e] della riduzione delle differenze non giustificate tra i redditi delle diverse discipline mediche». Dopo le spiegazioni da parte di chi ha presentato la proposta e la risposta del presidente H. H. Brunner, questa proposta è accettata tacitamente dai delegati.

Secondo un'altra proposta (n. 3 al punto 11.3) della SSMG, della SSML, della SSP e del Collegio di medicina di famiglia "la cifra 21 delle interpretazioni generali della versione TarMed alfa 2.2 (o alla cifra 85 a seconda delle versione) [deve essere] modificata imperativamentem. La proposta, troppo dettagliata per essere citata qui interamente, mira in sostanza a mantenere la possibilità di fatturare in più certi materiali d'uso corrente negli studi dei medici di famiglia, senza di che numerose prestazioni utili e poco costose sarebbero fornite solamente in ospedale. Gli esempi precisi di queste aberrazioni potrebbero essere comunicati a chi di diritto.

La questione è importante, i delegati che intervengono da parte delle società fanno capire che il mantenimento dell'interpretazione criticata è di natura tale da compromettere un'accettazione della TarMed in votazione generale. Agli atti.

\subsection{Convenzione quadro CAMS-FMH}

Dopo che il dott. A. Haefeli, presidente del «G 7» ha ricordato le attività di questo gruppo di lavoro e i risultati intermedi già ottenuti, e dopo un dibattito largamente utilizzato, la Camera medica, conformemente alla proposta del CC, prende conoscenza e approva tacitamente la convenzione quadro CAMSFMH e attribuisce alla delegazione alle negoziazioni l'incarico di proseguire le trattative sino alla loro con- 
clusione, basandosi sulle conclusioni e sulle decisioni della prossima seduta della Camera medica. Si noterà ancora, su domanda espressa del dott. L. Dubs, della Società svizzera d'ortopedia che la sigla EBM utilizzata in questo contesto non designa l'Evidence-based Medecine, ma le «Einheitliche Bemessungsmassstäbe». Agli atti.

\subsection{Concetto di neutralità dei costi CAMS-FMH} La proposta del CC su questo oggetto tende a far approvare dalla Camera il concetto di neutralità dei costi (qui di seguito: NC) CAMS-FMH, integrando, se necessario, le decisioni della Camera medica, con mandato alla nostra delegazione alle negoziazioni di condurre le trattative sino alla loro conclusione e di presentare il progetto definitivo del suddetto concetto in occasione della prossima seduta della Camera medica. Numerose proposte sono state presentata su questo oggetto. La prima (n. 2 al punto 11.5) che è stata presentata dal dott. R. Streit, per la Società dei medici del canton Berna, comprende 5 punti. Il punto 1 costata che la NC suddivisa in settori parziali (cantonale, ambulatorio, ambulatorio ospedale e ospedaliero) che dovrebbero essere neutrali in termini di costi non è conforme alla tariffa d'economia aziendale prescritta per legge. Si tratta di indicarlo chiaramente. Il punto 2 esige che la NC sia definita e limitata chiaramente. Il termine NC significa che i costi per assicurato non devono essere modificati dall'introduzione della struttura tariffaria. Tutte le influenze non strutturali che hanno un effetto sulla tariffa devono, nei limiti del possibile, essere registrate e imputate positivamente o negativamente alla somma della NC. Il punto 3 concerne l'angolo alfa: definito come un valore medio nazionale, dovrà essere adattato nei cantoni visto che il trasferimento tra i settori neutrali in materia di costi possono variare da un cantone all'altro. Per quel che concerne il punto 4 domanda che la fase di regolazione che segue l'introduzione della TarMed sia limitata a 2 anni al massimo. Il punto 5 chiede, infine, che i concetti presentati siano rimaneggiati e valutati per quel che concerne la loro contraddizione. In votazione, $i$ punti 1 , 2, 3, e 5 sono accettati ogni volta a fortissima maggioranza. Per quel che concerne il punto 4, viene accettato per quel che concerne la NC propriamente detta, il presidente H. H. Brunner fa tuttavia osservare che la Camera medica aveva precedentemente accettato il principio di una fase di convergenza di 5 anni. La seconda proposta (n. 3 al punto 11.5), presentata pure dal dott. R. Streit (BE), in tre punti, ricorda che la Camera medica ha approvato la struttura tariffaria calcolata su un valore del punto tariffario di Fr. 1.- e che quindi un valore del punto di Fr. -.80 è esplicitamente rifiutato (punto 1), che la Consigliera federale R. Dreifuss, con la sua dichiarazione al Consiglio degli Stati sul valore del punto prima della pubblicazione del risultato delle negoziazioni, ha dato prova di un atteggiamento parziale nei confronti del corpo medico (punto 2) e che non ci dovrebbe essere approvazione definitiva dei risul- tati delle negoziazioni sino a quando non avrà ritirato quanto affermato (punto 3). In votazione questa proposta è accettata a fortissima maggioranza.

La terza proposta (n. 4 al punto 11.5) proviene dalla Società di medicina del canton Friburgo. Domanda in sostanza che la Camera medica accetti l'introduzione della TarMed solo quando il concetto di neutralità dei costi sarà firmato da tutte le parti interessate (CAMS, Commissione delle tariffe mediche LAA, CDS e $\mathrm{H}+$ ), perché è imperativo che sia utilizzato un solo metodo di calcolo e di analisi. Il presidente $\mathrm{H}$. H. Brunner prende atto di questa proposta e promette di difenderla nel corso delle negoziazioni. La proposta è quindi accettata tacitamente.

Una quarta proposta (n. 5 al punto 11.5) presentata dalla FMS è ritirata dai suoi autori a profitto della proposte dei dott. R. Streit e E. Steinmann. La proposta di quest'ultimo (n. 6 al punto 11.5), presentata a nome della Società di medicina del canton di Lucerna, basandosi sulle decisioni prese nel corso delle precedenti Camere mediche, chiede che il concetto di NC soddisfi i punti seguenti: lo scopo del concetto di NC è l'introduzione neutrale dal punto di vista dei costi della nuova tariffa. La fase della NC rigorosa deve quindi essere introdotta al minimo; l'angolo alfa deve essere definito sul fondo in modo tale che non diventi l'oggetto di ulteriori negoziazioni; il principio, lo scopo e il meccanismo della convergenza (che deve essere terminata al più tardi in 5 anni) devono essere regolati severamente nel concetto; le condizioni quadro per l'implementazione del concetto (lista delle analisi, tariffa di fisioterapia, regolamentazione della distribuzione dei medicinali, obbligo a contrarre, ecc.) costituiscono parte integrante del concetto; le vie di diritto, rispettivamente l'esigenza di una decisione federale urgente non rientrano nelle competenze dei partner contrattuali e non potrebbero quindi far parte del concetto. Il concetto, in generale e in dettaglio, deve quindi essere modificato per tener conto di queste esigenze. Il presidente $\mathrm{H}$. $\mathrm{H}$. Brunner insiste affinché le condizioni quadro che saranno la garanzia del successo del funzionamento di questo concetto siano enumerate in modo completo. Questo messaggio ai politici e indispensabile. Detto ciò, si deve opporre al passaggio concernente la decisione federale urgente, perché le cose sono già regolate nell'ultima versione del concetto (non ancora in possesso dei delegati).

Il presidente constata, per concludere questo punto, che la proposta del Dott. E. Steinmann è accettata tacitamente, con la precisazione apportata dal dott. H. H. Brunner stesso.

\subsection{Concetto concernente il valore intrinseco}

La Camera medica accetta la proposta del CC di approvare il concetto concernente il valore intrinseco, nella versione del 4.6.2000, quindi in una versione che non si basa sul RFF attuale. Il presidente $\mathrm{H}$. $\mathrm{H}$. Brunner precisa che niente si oppone tuttavia al fatto che si ridiscuta ulteriormente con le SDM sui criteri qualitativi d'attribuzione del valore intrinseco. 
11.7 Convenzione quadro AINF/AM/AI

La Camera medica accetta la proposta del CC di approvare la convenzione quadro AINF/AM/AI nella sua versione 4 .

11.8 Concetto di neutralità dei costi AINF/AM/AI Non viene ancora presa nessuna decisione su questo documento.

\subsection{Valore iniziale del punto tariffario AINF/AM/AI}

La Camera medica prende conoscenza del commento in proposito, ma rimane inteso che la decisione definitiva spetta alla prossima Camera medica. Il dott. B. Meister, della Società svizzera d'anestesiologia, domanda che si voti sulla sua proposta che tende a fissare il punto iniziale a Fr. 1.-. Alla votazione, la proposta del dott. B. Meister raccoglie 125 voti favorevoli, 3 contrari e 13 astensioni.

\subsection{Agenda per l'introduzione delle tariffe TarMed}

La Camera medica approva con 138 voti favorevoli, nessuna opposizione né astensione, l'agenda proposta dal CC, che prevede che le negoziazioni siano condotte in modo da permettere l'introduzione della tariffa AINF/AM/AI tra il 1 gennaio e il 1 luglio 2001. Si tratterà in particolare di negoziare $\mathrm{il} / \mathrm{i}$ valore/i iniziale/i del punto tariffario AINF/AM/AI entro fine agosto 2000. I lavori e le negoziazioni concernenti l'introduzione della TarMed nelle tariffe cantonali devono essere organizzate in modo da permettere un'introduzione regolamentare al 1 luglio 2001. Il CC elabora uno scenario di ricambio permanente, seguendo l'evoluzione della scena politica, un'introduzione al 1 gennaio 2001. Le convenzioni quadro AINF/AM/AI come le convenzioni quadro CAMS-FMH, con le loro appendici, devono essere sottoposte ad una Camera medica straordinaria per approvazione. Il CC fissa la data corrispondente in modo da garantire la possibilità di una votazione generale.

L'accettazione di questo calendario comporterà forse la convocazione di una Camera medica che non potrà essere preceduta, come lo prevede lo statuto, da una Conferenza dei presidenti da tenere prima. La Camera è informata dal presidente su questa sospensione dello statuto dettata dalla necessità. Viene costatato formalmente che nessuna opposizione viene posta contro questo modo di procedere.

\subsection{Votazione generale}

La Camera medica accetta, con 137 voti favorevoli, nessun voto contrario e nessuna astensione, la proposta del CC che prevede l'organizzazione di una votazione generale prima dell'entrata in vigore delle tariffe TarMed nel settore AINF/AM/AI come anche in quello della LAMal. Saranno oggetto della votazione generale: la struttura tariffaria TarMed alfa 3i, la convenzione quadro AINF/AM/AI, con le sue appendici e la convenzione quadro CAMS-FMH, con le sue appendici. La votazione generale, che avrà luogo solamente se tutti questi punti saranno potuti essere approvati dalla Camera medica, dovrà aver luogo direttamente dopo che la Camera si sarà pronunciata su questi punti.

\subsection{Trasferimento elettronico dei dati} Questo punto non dà luogo a nessuna decisione.

\section{Concetto PR della FMH}

Considerata l'ora tardiva, non si è potuto tenere una discussione su questo documento del presidente. Quest'ultimo invita i delegati a comunicargli le loro eventuali osservazioni e suggerimenti di cui saprà tener conto.

\section{Varie}

Da ricordare in questa rubrica:

- che la FMH non resta inattiva sul terreno dei "Call Centers» e farà conoscere la sua posizione in tempo opportuno.

- che l'ORDINE, tramite il dott. A. Sury, invita la Camera medica a riunirsi l'anno prossimo in Ticino, in occasione del centenario della FMH. Il presidente H. H. Brunner accetta positivamente quest'offerta che dovrà tuttavia essere ancora studiata.

Sono quasi le 17 quando la sessione è tolta dal presidente che sottolinea l'assiduità dei delegati nell'occuparsi di un ordine del giorno impressionante e che ringrazia tutte le collaboratrici e tutti i collaboratori della segreteria generale che hanno contribuito al buon svolgimento di queste due giornate di riunione, citando in particolare il servizio di traduzione e gli interpreti. 


\section{Facharztprüfung zur Erlangung des Facharzttitels FMH für Rheumatologie}

Aufgrund des Weiterbildungsprogrammes, welches am 1. Januar 1996 in Kraft gesetzt wurde, ist die Teilnahme an der Facharztprüfung für diejenigen Kandidatinnen und Kandidaten Voraussetzung für die Erlangung des Facharzttitels FMH für Rheumatologie, welche ihre Weiterbildung in Rheumatologie bis am 31. Dezember 1996 nicht abgeschlossen hatten. Es empfiehlt sich, die Facharztprüfung frühestens im letzten Jahr der reglementarischen Weiterbildung abzulegen (Art. 22 WBO).
Schriftliche Prüfung

Ort: Hotel Bern, Bern

Datum: Mittwoch, 17. Januar 2001

Prüfungsgebühr: Die Fachgesellschaft erhebt für die schriftliche Prüfung eine Prüfungsgebühr von Fr. 300.-.

Schriftliche Anmeldung:

Péter Tamás, Sekretariat SGR

c/o Schweiz. Rheumaliga

Renggerstrasse 71, Postfach, 8038 Zürich

Tel. 0148740 00/07, Fax 014874019

E-mail:pt@srl.ch

Anmeldefrist: 30. November 2000

\section{Facharztprüfung zur Erlangung des Untertitels Handchirurgie zu den Facharzttiteln FMH für Chirurgie, Kinderchirurgie, orthopädische Chirurgie sowie Plastische und Wiederherstellungschirurgie}

Aufgrund des Weiterbildungsprogrammes, welches am 1. Juli 1996 in Kraft gesetzt wurde, ist die Teilnahme an der Facharztprüfung für diejenigen Kandidatinnen und Kandidaten Voraussetzung für die Erlangung des Untertitels Handchirurgie zum Facharzttitel FMH für Plastische und Wiederherstel- lungschirurgie. Es empfiehlt sich, die Facharztprüfung frühestens im letzten Jahr der reglementarischen Weiterbildung abzulegen (Art. 22 WBO).

Ort und Zeitpunkt: Universitätsspital Zürich, Klinik für Wiederherstellungschirurgie, Rämistrasse 100 , 8091 Zürich

1. Termin: 24. November 2000

2. Termin: 6. April 2001

Prüfungsgebühr: Die Fachgesellschaft erhebt einen Unkostenbeitrag von CHF 500.-.

Anmeldung:

Dr. W. Künzi, Leitender Arzt

Universitätsspital Zürich

Klinik für Wiederherstellungschirurgie

Rämistrasse 100, 8091 Zürich

Tel. 0125527 07, Fax 012558948

E-mail doris.schreiber@chi.usz.ch

Anmeldefrist: 15. Oktober 2000 


\section{Examen de spécialiste en vue de l'obtention du titre de spécialiste FMH en rhumatologie}

Conformément au programme de formation postgraduée qui est entré en vigueur le $1^{\mathrm{er}}$ janvier 1996, la participation à l'examen de spécialiste est obligatoire pour les candidats à l'obtention du titre FMH en rhumatologie qui n'avaient pas terminé leur formation postgraduée en rhumatologie le 31 décembre 1996. Il est recommandé de passer l'examen de spécialiste au plus tôt durant la dernière année de la formation postgraduée réglementaire (art. 22 RFP).
Examen écrit

Lieu: Hôtel Bern, Berne

Date: Mercredi, 17 janvier 2001

Taxe d'examen: La société de discipline médicale prélève une participation aux frais de Fr. 300.- pour l'examen écrit.

Inscriptions (par écrit):

Péter Tamás, Secrétariat SSR

c/o Schweiz. Rheumaliga

Renggerstrasse 71, Case postale, 8038 Zurich

tél. 0148740 00/07, fax 014874019

e-mail:pt@srl.ch

Délais d'inscription: le 30 novembre 2000
Examen de spécialiste en vue de l'obtention du sous-titre en chirurgie de la main de spécialiste FMH en chirurgie, chirurgie pédiatrique, chirurgie orthopédique et chirurgie plastique et reconstructive

Conformément au programme de formation postgraduée entré en vigueur le $1^{\text {er }}$ juillet 1996, la participation à l'examen de spécialiste est une condition requise pour les candidats à l'obtention du sous-titre en chirurgie de la main de spécialiste FMH en chirurgie plastique et reconstructive. Il leur est recommandé de passer l'examen de spécialiste au plus tôt durant la dernière année de leur formation postgraduée réglementaire (art. 22 RFP).

Lieu et date: L'Hôpital universitaire, Clinique de Chirurgie reconstructive, Rämistrasse 100, 8091 Zurich

$1^{\text {re }}$ date: le 24 novembre 2000

$2^{\mathrm{e}}$ date: le 6 avril 2001

Taxe d'examen: La société de discipline médicale prélève une participation aux frais de CHF 500.-.

\section{Inscription:}

auprès du Dr W. Künzi

L'Hôpital universitaire

Clinique de Chirurgie reconstructive

Rämistrasse 100, 8091 Zurich

tél. 0125527 07, fax 012558948

e-mail doris.schreiber@chi.usz.ch

Délai d'inscription: le 15 octobre 2000 


\begin{tabular}{|c|c|c|}
\hline \multicolumn{3}{|c|}{ Managementgrundlagen für Ärztinnen und Ärzte } \\
\hline \multicolumn{3}{|l|}{ Daten } \\
\hline Element 1 & 15./16. September 2000 & 1,5 Tage \\
\hline Element 2 & $\begin{array}{l}\text { 30. November / } \\
\text { 1. Dezember } 2000\end{array}$ & 2 Tage \\
\hline Element 3 & 26./27. Januar 2001 & 1,5 Tage \\
\hline Element 4 & 23./24. März 2001 & 1,5 Tage \\
\hline
\end{tabular}

Verantwortliche Programmleiter

Peter Berchtold, College-M, Bern

Christof Schmitz, Corporate Pragmatics, Zürich

Susanne Quistorp, Zürich

Ort
1) Offenes Programm: Stadt Zürich
2) Programm für das Universitätsspital Zürich: Stadt Zürich
3) Programm für das Inselspital Bern: College-M, Bern

\section{Qualitätsmanagement}

Daten

Basisprogramm

2. Durchführung

$$
\text { 19.-21. Oktober } 2000 \quad \text { 2,5 Tage }
$$

\section{Aufbauprogramm}

Element 1 23.-25. November $2000 \quad 3$ Tage

Element 2 11.-13. Januar 2001 Tage

Verantwortliche Programmleiter Peter Berchtold, College-M, Bern Hans Heinrich Brunner, FMH, Bern Richard Cranovsky, FMH, Bern

Ort: Stadt Bern

Kosten: Basisprogramm Fr. 1050.Aufbauprogramm Fr. 1850.-
Studienreise in den englischen National Health Service (NHS): Neue Perspektiven für Gesundheitssysteme

Daten: 16.-21. Oktober 2000

Ort: London und Glasgow

Programmleiter

Peter Berchtold, College-M, Bern

Andy Kennedy, King’s Fund, London

Kosten: Fr. 3800.- inkl. Hotel und Verpflegung, exkl. Reisekosten

\section{Nachdiplomstudium «Managed Care»}

\section{Daten}

Einführung in das NDS

26. -28. Oktober 2000

\section{Modul 1}

Versorgungsqualität und Patientenprozesse

Block 1 13.-17. November 2000

Block 2 14.-16. Dezember 2000

Block 3 11.-13. Januar 2001

\section{Managementprogramm FE2}

Daten

$\begin{array}{lll}\text { Element 1 } & \text { 16.-18. Oktober 2000 } & \text { 3 Tage } \\ \text { Element 2 } & \text { 8.-10. November 2000 } & \text { 3 Tage } \\ \text { Element 3 } & \text { 13.-16. Dezember 2000 } & \text { 4 Tage } \\ \text { Element 4 } & \text { 18.-20. Januar 2001 } & \text { 3 Tage } \\ \text { Element 5 } & \text { 28. Februar-2. März 2001 } & \text { 3 Tage } \\ \text { Element 6 } & \text { 5.-6. April 2001 } & \text { 2 Tage }\end{array}$

Verantwortliche Programmleiter

Peter Berchtold, College-M, Bern

Christof Schmitz, Corporate Pragmatics, Zürich

Ort: College-M, Bern

Kosten: Fr. 7920.-

\section{Name}

\section{Informationsbestellschein}

Bitte senden Sie mir Informationen zu den folgenden Programmen:

$\square$ Managementgrundlagen für Ärztinnen und Ärzte

$\square$ Qualitätsmanagement

$\square$ Studienreise NHS

$\square$ Nachdiplomstudium «Managed Care»

$\square$ Managementprogramm FE2

Vorname
Institution
Adresse
PLZ / Ort
Telefon
E-mail

College-M, Postfach 293, 3000 Bern 16, Tel. 031359 11 37, Fax 03135911 12, E-mail info@college-m.ch 